\section{Alimentos orgânicos e saúde humana: estudo sobre as controvérsias}

\author{
Anete Araújo de Sousa, ${ }^{1}$ \\ Elaine de Azevedo, ${ }^{2}$ \\ Elinete Eliete de Lima ${ }^{1}$ \\ e Ana Paula Ferreira da Silva ${ }^{1}$
}

Como citar: Sousa AA, Azevedo E, Lima EE, Silva APF. Alimentos orgânicos e saúde humana: estudo sobre as controvérsias. Rev Panam Salud Publica. 2012;31(6):513-7.

\section{SINOPSE}

O estudo das controvérsias é uma ferramenta metodológica para o conhecimento das dimensões sociais e políticas da ciência. O tema dos alimentos orgânicos pode ser compreendido e explorado a partir dessa abordagem. $O$ objetivo deste artigo foi analisar as controvérsias referentes ao status do alimento orgânico. A pesquisa foi realizada com base em referências a partir de 1990, citadas nos sites da International Foundation for Organic Agriculture, da Soil Association $e$ da Food and Agriculture Organization. Foram identificadas controvérsias acerca 1) das repercussões sobre a saúde humana da existência de contaminantes químicos nos alimentos orgânicos; 2) da qualidade dos alimentos orgânicos em comparação aos convencionais; e 3) da temática referente ao preço dos alimentos orgânicos. O artigo conclui que, embora os alimentos orgânicos se destaquem por sua baixa toxicidade, maior durabilidade e teor de alguns nutrientes em alguns alimentos, mais estudos comparativos devem ser realizados para comprovar a superioridade do seu valor nutricional e para que as controvérsias se dissolvam. É preciso contextualizar a discussão em um amplo espectro de promoção da saúde, no qual a produção orgânica aparece vinculada ao fomento ao pequeno agricultor, à biodiversidade e ao desenvolvimento local sustentável, de modo a garantir o aumento da demanda e da oferta de produtos orgânicos a preços justos para consumidores individuais e institucionais.

Palavras-chave: alimentos orgânicos; contaminantes químicos em alimentos; ecologia da nutrição; qualidade dos alimentos; saúde ambiental.

1 Universidade Federal de Santa Catarina, Centro de Ciências da Saúde, Programa de Pós-Graduação em Nutrição, Núcleo de Pesquisa de Nutrição em Produção de Refeições (NUPPRE), Florianópolis (SC), Brasil. Correspondência: anete@ccs.ufsc.br (Anete Araújo de Sousa).

2 Universidade Federal da Grande Dourados (UFGD), Faculdade de Ciências da Saúde, Dourados (MG), Brasil.
O estudo das controvérsias é uma ferramenta metodológica para o conhecimento das dimensões sociais e políticas da ciência. Nesse campo, é possível aprender sobre as dinâmicas da produção científica e tecnológica em suas relações com a sociedade (1).

A análise de pesquisas na área de alimentação e nutrição revela evidências contraditórias acerca da segurança e da eficácia de práticas de uso dos alimentos, nutrientes ou suplementos. A cada dia surgem novos estudos que questionam os anteriores. Algumas pesquisas, descritas a seguir e analisadas por Azevedo (2), ilustram essas contradições.

O consumo de ovos, por exemplo, foi restrito para prevenir riscos de doença cardiovascular. Evidências científicas posteriores indicaram, porém, uma associação fraca entre a restrição de ovos e a redução dos riscos de doença cardiovascular e derrame $(3,4)$. O consumo de café também já foi relacionado à etiologia da hipertensão. No entanto, outro estudo apontou que, embora o consumo de café esteja associado a pequenas alterações na pressão sanguínea, ele não tem um papel central no aparecimento da hipertensão (5).

Também serve de exemplo o caso da dieta rica em vegetais, frutas e grãos, preconizada pelos médicos e nutricionistas para o controle de doenças cardiovasculares. Um estudo com mais de $48000 \mathrm{mu}-$ lheres não reduziu significativamente o risco de tais doenças nessa população (6). Foi demonstrado ainda que nem todas as gorduras são nocivas e que existem também "gorduras boas". As nozes, antes consideradas prejudiciais por seu alto conteúdo de gordura, são atualmente relacionadas à prevenção de doenças cardíacas. O consumo de chocolate, já associado à obesidade, pode fazer bem à saúde. Finalmente, pesquisadores apontam que substâncias chamadas flavonoides, encontradas no cacau, podem diminuir o colesterol (7). A abordagem dessas controvérsias científicas pode auxiliar na compreensão do tema dos alimentos orgânicos.

Os alimentos orgânicos são definidos como aqueles alimentos in natura ou processados que são oriundos de um sistema orgânico de produção agropecuária e industrial. A produção de alimentos orgânicos é baseada em técnicas que dispensam o uso de insumos como pesticidas sintéticos, fertilizantes químicos, medicamentos veterinários, organismos geneticamente modificados, conservantes, aditivos e irradiação. A ênfase da produção está direcionada ao uso de práticas de gestão e manejo do solo que levam em conta as condições regionais e a necessidade de adaptar localmente os sistemas de produção (8). É importante destacar que, mesmo que a produção dos alimentos orgânicos não utilize esses insumos, não é possível garantir a ausência total de resíduos de con- 
taminantes químicos, por problemas relacionados à contaminação ambiental com produtos persistentes e também por derivação e proximidade de propriedades convencionais (9).

As restrições quanto aos diferentes contaminantes se devem aos diversos (e controversos) estudos que apresentam efeitos de algumas dessas substâncias na saúde humana. Outra questão diz respeito ao declínio da qualidade do solo e da quantidade de nutrientes, especialmente micronutrientes, em muitos alimentos provenientes dos métodos convencionais de plantio, irrigação e uso intensivo de agrotóxicos e fertilizantes $(10,11)$. Também há controvérsia quanto ao valor nutricional e ao preço de venda dos alimentos orgânicos em comparação aos alimentos produzidos convencionalmente.

$\mathrm{O}$ objetivo do presente artigo foi analisar as controvérsias referentes ao status do alimento orgânico. Foram abordadas as repercussões sobre a saúde humana da existência de contaminantes químicos nesses alimentos, a qualidade dos alimentos orgânicos em comparação com os convencionais e a temática referente ao preço dos alimentos orgânicos. A pesquisa baseou-se em referências a partir de 1990, citadas nos sites International Foundation for Organic Agriculture (http://www.ifoam.org/), Soil Association (http:// www.soilassociation.org/) e Food and Agriculture Organization (www.fao.org).

\section{CONTAMINANTES QUÍMICOS E A SAÚDE HUMANA}

Não há informações suficientes e seguras sobre o poder cumulativo, o efeito combinado, a mutabilidade (capacidade de sofrer mudanças em seu nível de toxicidade após a ingestão) e as possibilidades de interação no organismo humano de muitos contaminantes utilizados no sistema agroalimentar (12). Portanto, não é possível estabelecer inter-relações precisas e imediatas entre as consequências do consumo dessas substâncias em longo prazo e as diferentes enfermidades. Além disso, essas substâncias são, muitas vezes, ofertadas em doses acima das recomendadas e sem controle adequado por parte dos sistemas de vigilância.

A maioria dos países adota sistemas de avaliação para estimar, cientificamente, o risco potencial para a saúde humana da presença de substâncias químicas em alimentos. As abordagens de gestão de risco variam dependendo da origem do produto químico: adicionado intencionalmente ao alimento ou resultado da contaminação acidental. Para a Organização das Nações Unidas para Agricultura e Alimentação (FAO) e a Organização Mundial da Saúde (OMS), a avaliação da exposição deveria ser ampliada de forma a considerar as diferenças nos hábitos alimentares entre os países. Essas organizações recomendam ainda que os países realizem análises baseadas no estudo da dieta total (EDT) para avaliar a exposição da população em geral e de grupos vulneráveis, como as crianças, a contaminantes químicos. O método EDT estima a in- gestão dietética de elementos químicos e de nutrientes através de análises diretas em amostras de alimentos preparados que reflitam os hábitos dietéticos médios de grupos populacionais $(13,14)$.

Azevedo e Rigon (15) abordam diferentes estudos que apresentam efeitos dos agrotóxicos sobre a saúde humana, tais como imunodepressão, mal de Parkinson, depressão e outras desordens neurológicas, aborto e problemas congênitos, alguns tipos de câncer (especialmente os hormônio-dependentes), infertilidade, má formação congênita, sintomas respiratórios e esterilidade em adultos. As autoras também compilam estudos que sinalizam manifestações clínicas (rinite, urticária, angioedema, asma e alergias) provocadas pelos aditivos químicos sintéticos, em particular pelos corantes artificiais.

Estudos citados por Powlson al. (16) mostram uma associação positiva entre nitrato e linfomas não Hodgkin, câncer de bexiga, ovário, útero e colo retal e um tipo de anemia em bebês, a metaemoglobinemia. Entretanto, esses autores relatam efeitos benéficos dos nitratos em gastrenterites e doenças cardiovasculares. Tais controvérsias sugerem a necessidade de mais estudos que esclareçam a real dimensão de cada substância sobre a saúde humana.

As repercussões acima mencionadas são quantitativamente modestas diante do número de substâncias usadas no sistema agroalimentar convencional. Os efeitos de outros contaminantes precisam ser mais bem delineados; também são necessários mais estudos que avaliem os efeitos das tecnologias sobre a saúde humana, entre eles a irradiação de alimentos, a transgenia e a nanotecnologia.

Destaca-se a complexidade de se analisar contaminantes químicos nos alimentos e a dificuldade de relacionar tais substâncias à etiologia de enfermidades. Por isso, as legislações de alimentos orgânicos consideram que, diante de um possível perigo à saúde, a substância ou a tecnologia deve ser evitada, respeitando-se o princípio da precaução (17), que tem sido tomado como referência em muitas discussões que envolvem riscos.

\section{ALIMENTOS ORGÂNICOS FRENTE A ALIMENTOS CONVENCIONAIS}

Na literatura científica, algumas pesquisas avaliaram os benefícios do consumo de alimentos orgânicos para a saúde humana. Tais estudos alegam que uma dieta orgânica pode diminuir a exposição de crianças aos pesticidas $(18,19)$ e apresentar efeito positivo no quesito fertilidade, uma vez que muitos pesticidas são disruptores endócrinos (uma dieta isenta dessa classe de agrotóxicos pode ter um efeito sobre a fertilidade masculina) $(20,21)$. No entanto, como mencionado anteriormente, é difícil estabelecer relações, pois os estudos populacionais que compararam a saúde das pessoas que consomem habitualmente alimentos orgânicos com a saúde daquelas que consomem alimentos convencionais apresentaram grande número de variáveis não controladas. 
Quanto às comparações sobre valor nutricional, muitos fatores e variáveis devem ser considerados nas pesquisas, tais como o tempo de produção orgânica, o restabelecimento da vida do solo, o tipo de sistema orgânico utilizado, a variabilidade dos fatores externos (luz solar, temperatura, chuva), o armazenamento e o transporte, que influenciam diretamente o conteúdo de nutrientes nas plantas (22). O desempenho de sistemas produtivos orgânicos e convencionais deve ser estudado na propriedade de origem (23), onde o grau de controle dos fatores externos supramencionados é menor do que nos laboratórios. Assim, é possível perceber a dificuldade de planejar estudos efetivos, cujos resultados possam ser sistematizados e comparados aos de diferentes pesquisas.

Os resultados dos estudos que compararam os alimentos orgânicos e os convencionais foram sintetizados em duas grandes revisões realizadas em 2009. Um delas se posiciona claramente contra a superioridade dos orgânicos em termos nutricionais (24); a outra é mais favorável (25), mas ainda assim sinaliza controvérsias no campo de estudo. Pesquisadores da Food Standards Agency (FSA), do Reino Unido, afirmam não haver evidências de benefícios para a saúde no consumo dos alimentos orgânicos comparados aos convencionais em relação ao valor nutricional. Por isso, atestam que tais alimentos não são de relevância para a saúde pública (24).

Por outro lado, a Agence Française de Sécurité Sanitaire des Aliments (AFSSA) realizou uma avaliação de estudos sobre qualidade nutricional dos alimentos orgânicos comparados aos convencionais e encontrou resultados opostos: maior teor de matéria seca em tubérculos, raízes e folhas; maior teor de ferro e magnésio em vegetais como batata, couve, cenoura, beterraba, alho-poró, alface, cebola, aipo e tomate; mais vitamina $\mathrm{C}$ na batata, alho-poró, couve e aipo; maiores quantidades de betacaroteno no tomate, cenoura e leite orgânicos; maiores quantidades de fitoquímicos na maçã, pêssego, pera, laranja, cebola, tomate, batata, pimentão, óleo de oliva (compostos fenólicos), vinho (resveratrol) e tomate (ácido salicílico) (25). O estudo francês destaca ainda o maior teor de ácidos graxos poli-insaturados no leite, ovos e carnes orgânicas, uma vez que a dieta à base de pasto e a criação livre preconizada no manejo animal orgânico têm como resultado carne e leite com menores teores de gordura saturada (25). Ambas as revisões confirmam o teor aumentado de nitratos em alimentos de origem convencional.

Quanto aos aspectos sensoriais, embora faltem evidências conclusivas, há indicações de que os alimentos orgânicos sejam mais saborosos (26). Contudo, a análise dos aspectos sensoriais de qualidade é complexa, uma vez que é subjetiva a característica de um alimento que determina a aceitabilidade do consumidor (9). Outro aspecto importante relaciona-se à durabilidade, uma vez que a adubação à base de nitrogênio utilizada na agricultura convencional promove um aumento no teor de água dos vegetais, tornando tais alimentos mais perecíveis (22).
A utilização de dejetos de animais pelo sistema orgânico na horticultura levanta suspeitas sobre sua qualidade microbiológica e parasitária. Entretanto, seguindo-se boas práticas agrícolas que minimizem os riscos de contaminação biológica, não há evidências de que os orgânicos sejam mais suscetíveis à contaminação microbiológica quando comparados aos sistemas convencionais (26). Com relação às micotoxinas (toxinas produzidas por fungos), a FAO (9) ressalta não haver comprovação de que os alimentos orgânicos sejam mais contaminados. A revisão da AFSSA (25) destaca que é semelhante o teor de micotoxinas nos cereais orgânicos e nos convencionais.

Quanto ao preço dos alimentos orgânicos, Azevedo (22) destaca as variantes envolvidas no processo produtivo dos alimentos. De forma simplificada, alega-se que o valor agregado, que pode variar de 20 até $100 \%$ mais para os produtos orgânicos em relação aos de origem convencional, tem como uma das causas a lei da oferta e da procura. Frente à baixa demanda, quando comparado ao alimento convencional, o produto orgânico ainda não é competitivo no grande mercado. Entretanto, outros aspectos relativos à comercialização precisam ser analisados no sentido de impulsionar a comercialização dos orgânicos, já que o preço dificulta a acessibilidade. É preciso, entre outros, entender o confronto entre o grande circuito de comercialização (o de supermercados) e os circuitos curtos (de feiras e venda direta).

O grande circuito impõe ao agricultor barreiras como a padronização e a incorporação de serviços aos produtos (uso de embalagens plásticas ou isopor), contratos regulares de entrega (nem sempre possíveis em função da sazonalidade dos alimentos in natura) e a não remuneração do produto não comercializado, entre outras. Além disso, faz uso de margens altas para o aumento da lucratividade, o que dificulta a venda e elitiza o consumo de alimentos orgânicos. Por outro lado, o supermercado permite que uma fatia de consumidores urbanos descubra o produto orgânico, tornando-o mais conhecido e acessível.

A venda direta e as feiras são propostas eficazes para o fortalecimento de associações de agricultores orgânicos. Porém, há dificuldades, como a distância dos centros consumidores, as condições das estradas e a exigência tanto de habilidade para o comércio quanto de tempo disponível do agricultor para a venda. Destaca-se que esse circuito é voltado para um consumidor já sensibilizado para o consumo e a compra de alimentos orgânicos de produção local, havendo alguma dificuldade de ampliar o número de envolvidos. Por outro lado, as vendas diretas promovem um estreitamento com os consumidores, fidelizando-os cada vez mais à proposta da agricultura orgânica e sustentável. Além disso, a ausência de intermediação permite uma maior apropriação, pelos agricultores, dos resultados de seu trabalho, em termos de renda.

A produção orgânica exige maior envolvimento de mão de obra. Ao adquirir esse tipo de alimento, o consumidor passa a contribuir para o fortalecimento e a viabilidade da agricultura familiar. Essa é uma con- 
tribuição social do consumidor socioambientalmente consciente: ao buscar produtos orgânicos, ele assume um papel decisivo nesse contexto de transição.

O alimento é uma mercadoria que o consumidor exige que tenha preço baixo e alta qualidade - e o preço baixo de um alimento ou refeição raramente leva em conta o custo ambiental, os gastos energéticos para sua produção, os impactos na saúde humana, no bem-estar animal e na qualidade de vida dos que produzem tais alimentos. Ao adquirir o alimento orgânico, o consumidor contribui para a promoção da sua saúde, para a qualidade de vida das futuras gerações e para a preservação dos ecossistemas naturais. Nesse contexto, a pergunta que deve ser feita é: qual o real valor de um alimento com preço baixo, mas que promove a poluição ambiental, a perda da biodiversidade, a exclusão social e que contribui para o aumento das doenças?

\section{CONSIDERAÇÕES FINAIS}

Com base nos estudos analisados, concluímos que, embora os alimentos orgânicos se destaquem por sua baixa toxicidade, maior durabilidade e maior teor de alguns nutrientes em alguns alimentos, mais estudos comparativos devem ser realizados para comprovar essa superioridade e determinar se existem vantagens em termos de valor nutricional para que as controvérsias se dissolvam. Por outro lado, é preciso considerar um contexto de saúde ampliado, que não se resuma a uma análise do valor nutricional desses alimentos.

Sem desconsiderar o foco do artigo e as controvérsias analisadas, destaca-se que, ao optar por alimentos orgânicos, o consumidor está ingerindo menos substâncias tóxicas e apoiando um processo de transição ecológica que visa à desintoxicação gradual dos alimentos, do solo e das águas, promovendo a saúde ambiental. Portanto, o amplo espectro de promoção da saúde ao qual o sistema orgânico aparece vinculado, especialmente o fomento ao pequeno agricultor, à bio- diversidade e ao desenvolvimento local sustentável, é um apelo para que esse tipo de produção seja estimulado e subsidiado mundialmente, de modo a garantir o aumento da sua demanda e da oferta com preços justos para consumidores individuais e institucionais.

\section{SYNOPSIS}

\section{Organic foods and human health: a study of controversies}

The study of controversies is a methodological tool that generates knowledge about the social and political dimensions of science. This approach can be used to understand and explore the topic of organic foods. The present study aimed to analyze the controversies regarding the status of organic foods. We carried out a review of studies published since 1990 in three websites: International Foundation for Organic Agriculture, Soil Association, and Food and Agriculture Organization. The following controversies were identified: 1) effects on human health of the presence of chemical contaminants in organic foods; 2) the quality of organic foods as compared to conventionally grown foods; and 3) price of organic foods. Based on this review, it is possible to conclude that, even though organic foods stand out for their low toxicity, higher durability, and nutritional content of some items, more comparative studies are required to confirm the nutritional superiority of organic foods and to solve the controversies. The discussion must be contextualized within a broad spectrum of health promotion, in which organic farming appears associated with the support for small farming, biodiversity, and local sustainable development, so as to increase offer and demand for organic products at fair prices for individual and institutional consumers.

Key words: food, organic; food pollutants, chemical; nutrition ecology; food quality; environmental health.

\section{REFERÊNCIAS}

1. Pinch $Y$, Leunberger C. Studying scientific controversy from the STS perspective. 2006. Department of Science \& Technology Studies, Cornell University. Disponível em: http://ionesco. sciences-po.fr/com/moodledata/3/ Pinch_Leuenberger_Controversies.pdf Acessado em 5 de novembro de 2009.

2. Azevedo E. Riscos e controvérsias no processo de construção do conceito de alimento saudável: o caso da soja [tese]. Florianópolis: Universidade Federal de Santa Catarina; 2009.

3. Hu FB, Stampfer MJ, Rimm EB, Manson JE, Ascherio A, Colditz GA, et al. A prospective study of egg consumption and risk of cardiovascular disease in men and women. JAMA. 1999;281(15):1387-9.
4. Dawber TR, Nikcerson RJ, Brand FN, Pool J. Eggs, serum cholesterol, and coronary heart disease. Am J Clinical Nutr. 1982;36(4):617-25.

5. Klag MJ, Wang NY, Meoni LA, Brancati FL, Cooper LA, Liang KY, et al. Coffee intake and risk of hypertension: The Johns Hopkins Precursors Study. Arch Intern Med. 2002;162(6):657-62.

6. Howard B, Van Horn L, Hsia J, Manson JE, Stefanick ML, Wassertheil-Smoller S, et al. Low-fat dietary pattern and risk of cardiovascular disease: the Women's Health Initiative Randomized Controlled Dietary Modification Trial. JAMA. 2006;295(6):655-66.

7. Hu F, Stampher M, Manson J, Rimm EB, Colditz GA, Rosner BA, et al.
Frequent nut consumption and risk of coronary heart disease in women: prospective cohort study. BMJ. 1998; 317(7169):1341-5

8. Food and Agriculture Organization, FAO Inter-Departmental Working Group on Organic Agriculture. Organic agriculture. Disponível em: http://www. fao.org/organicag/oa-faq/oa-faq1/es/ Acessado em 8 de março de 2012.

9. Food and Agriculture Organization. Influence de L'Agriculture Biologique sur L'Innocuité et la Qualité des Aliments. Vingt-Deuxième Conférence Régionale de la FAO pour L'Europe; 2000 Jul 24-28; Porto, Portugal. Disponível em: http:// www.fao.org/docrep/meeting/X4983F. $\mathrm{htm}$ Acessado em 5 de maio de 2003. 
10. Davis D, Epp MD, Riordan HD. Changes in USDA food composition data for 43 garden crops, 1950 to 1999. J Am Coll Nutr. 2004;23(6):669-82.

11. White PJ, Broadley MR. Historical variation in the mineral composition of edible horticultural products. J Hortic Sci Biotech. 2005;80(6):660-7.

12. Roe F. Some aspects of food toxicology: a personal view. Em: Miller K. Toxicological aspects of food. Londres: Elsevier; 1997.

13. Organización de las Naciones Unidas para la Agricultura y la Alimentación. Conferencia Paneuropea de FAO/OMS sobre Inocuidad y Calidad Alimentaria; 2002 Feb 25-28; Budapeste, Hungria. Disponível em: ftp://ftp.fao.org/ docrep/fao/meeting/004/x6865s.pdf Acessado em 15 de março de 2012.

14. Food and Drug Administration. Total Diet Study. Disponível em: http:// www.fda.gov/Food/FoodSafety/Food ContaminantsAdulteration/TotalDiet Study/default.htm Acessado em 23 de março de 2012.

15. Azevedo E, Rigon SA. Sistema alimentar com base no conceito de sustentabilidade. Em: Taddei JA, Lang RMF, LongoSilva G, Toloni MHA, eds. Nutrição em saúde pública. São Paulo: Rubio; 2010. Pp. 543-60.

16. Powlson DS, Addiscott TM, Benjamin N, Cassman KG, Kok TM, Grinsven H, et al. When does nitrate become a risk for humans? J Environ Qual. 2008;37:291-5.

17. Wynne B. Uncertainty and environmental learning: reconceiving science and policy in the preventive paradigm. Glob Environ Change. 1992;2(2):111-27.

18. Curl CL, Fenske RA, Elgethun K. Organophosphorus pesticide exposure of urban and suburban pre-school children with organic and conventional diets. Environ Health Perspect. 2003;111(3):377-82.

19. Lu C, Toepel $K$, Irish R, Fenske RA, Barr DB, Bravo R. Organic diets significantly lower children's dietary exposure to organophosphorus pesticides. Environ Health Perspect. 2006;114(2):260-3.

20. Abell A, Ernst E, Bonde JP. High sperm density among members of organic farmers' association. Lancet. 1994;343 (8911):1498.

21. Jensen TK, Giwercman A, Carlsen E, Scheike T, Skakkebaek NE. Semen quality among members of organic food associations in Zealand, Denmark. Lancet. 1996;347(9018):1844.
22. Azevedo E. Alimentos orgânicos: ampliando conceitos de saúde humana, social e ambiental. Tubarão: Unisul; 2006.

23. Benbrook $\mathrm{CM}$, Zin Zhao $X$, Yáñez J, Davies N, Preston A. New evidence confirms the nutritional superiority of plant-based organic foods. Boulder: The Organic Centre; 2008. Disponível em: http://www.organiccenter.org/reportfiles/5367_Nutrient_Content_SSR FINAL_V2.pdf Acessado em 8 de março de 2008.

24. Dangour AD, Dodhia SK, Hayter A, Allen E, Lock K, Uauy R. Nutritional quality of organic foods: a systemic review. Am J Clin Nutr. 2009;90(3):680-5.

25. Lairon D. Nutritional quality and safety of organic food. A review. Agron Sustain Dev. 2009;30(1):33-41.

26. Bourn D, Prescott J. A comparison of the nutritional value, sensory qualities, and food safety of organically and conventionally produced foods. Crit Rev Food Sci Nutr. 2002;42(1):1-34.

Manuscrito recebido em 20 de julho de 2011 Aceito em versão revisada em 3 de abril de 2012. 